\title{
Corneal vesicles accumulate collagen VI associated with tissue remodeling in apolipoprotein a-I deficiency: a case report
}

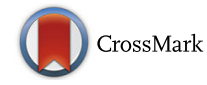

\author{
Hiroyuki Namba ${ }^{1 *} \mathbb{D}$, Mari Narumi ${ }^{1}$, Shinji Susa ${ }^{2}$, Rintaro Ohe $^{3}$, Takeo Kato², Mitsunori Yamakawa ${ }^{3}$ \\ and Hidetoshi Yamashita ${ }^{1}$
}

\begin{abstract}
Background: Apo A-I deficiency clinically shows low serum levels of HDL cholesterol and corneal opacity at a young age. Histopathological evaluations of affected corneas are not enough, and the mechanism of corneal opacity is still unclear.
\end{abstract}

Case presentation: A 61-year-old woman suffered from blurred vision with a corneal opacity. She had significantly reduced serum levels of high-density lipoprotein cholesterol and Apo A-I, stenosis of the coronary arteries, and ischemic heart failure. On genetic examination, a homozygous mutation of Apo A-I $\left.\right|_{\text {Tsukuba }}$ was identified. Histopathological examination of the corneal button after PKP showed numerous vesicles in the corneal stroma, which were more prominent in the deep stroma than in the shallow stroma. Collagen VI was observed in some of those vesicles.

Conclusion: We experienced a rare case of corneal opacity due to Apo A-I deficiency. Our histopathological findings indicated that structural changes in corneal collagen fibrils contribute to the formation of stromal vesicles.

Keywords: Case report, Apolipoprotein a-I deficiency, Collagen VI, Confocal microscopy, Corneal opacity, Immunohistochemistry

\section{Background}

Apo A-I is an essential component of HDL. Its deficiency is clinically detected by low serum levels of HDL cholesterol. Although different gene mutations of Apo A-I are reported [1-4], the typical clinical presentation includes corneal opacity, xanthoma, and ischemic heart disease at a young age. Histopathological evaluations of affected corneas are not enough, and the mechanism of corneal opacity is still unknown. In the present study, we report a part of the mechanism associated with corneal opacity in a case of genetic Apo A-I deficiency.

\section{Case presentation}

In 2012, a 61-year-old woman with blurred vision presented with corneal opacity of the left eye. Her visual acuity was 20/40 OS and 20/20 OD. She had been

\footnotetext{
* Correspondence: h-nanba@med.id.yamagata-u.ac.jp

${ }^{1}$ Department of Ophthalmology and Visual Sciences, Yamagata University

Faculty of Medicine, 2-2-2 lidanishi, 9909585 Yamagata City, Yamagata, Japan

Full list of author information is available at the end of the article
}

diagnosed with HDL deficiency at the age of 46 years, 1997. but she had been off treatment for about 10 years. She had undergone PKP of the right eye for the same symptoms when she was 58 years old, 2009.

The patient was the fourth of 8 siblings. A brother died in an accident and a sister died in childhood. None of her family members had been diagnosed with HDL deficiency, and there was no family history of consanguineous marriage. On physical examination, she had bilateral xanthomas of the eyelids and focal yellow skin discolorations in the cubital fossae of both forearms, but there was no tonsillar hypertrophy or Achilles tendon xanthoma.

Blood tests showed an extremely low HDL-cholesterol level $(6 \mathrm{mg} / \mathrm{dL})$ with undetectable Apo A-I $(<5 \mathrm{mg} / \mathrm{dL})$. Levels of Apo B and Apo E were slightly elevated $(109 \mathrm{mg} / \mathrm{dL}$ and $5.8 \mathrm{mg} / \mathrm{dL}$, respectively). Serum triglyceride, total cholesterol, low-density lipoprotein cholesterol, and lecithin-cholesterol acyltransferase levels were within the normal limits. A genetic evaluation was performed as 
previously described [4], and showed that she was homozygous for the Apo A- $\mathrm{I}_{\text {Tsukuba }}$ mutation, which is a single-nucleotide insertion of cytosine that alters the reading frame from codon 5 and produces a premature stop codon at codon 34 (Fig. 1). Thus, this patient was diagnosed with genetic Apo A-I deficiency. Electrocardiogram, echocardiogram, and myocardial scintigraphy showed significant ischemic heart failure and angiography showed severe stenosis of the coronary arteries. Consequently, she underwent percutaneous coronary intervention and coronary artery bypass grafting.

On slit-lamp examination, there was a diffuse corneal stromal opacity with a lucid zone in the left eye. Confocal microscopy (Heidelberg Retinal Tomograph Rostock Cornea Module, Heidelberg Engineering, Heidelberg, Germany) revealed high-intensity vesicles $(10-100 \mu \mathrm{m}$ in diameter) in the corneal stroma (Fig. 2). In 2013, PKP was performed for the left eye, and the corneal button tissues were examined histopathologically and immunohistochemically.
Histopathological and immunohistochemical analysis of the corneal button

The cornea showed normal thickness and there was no significant abnormality of the epithelium, Bowman's layer, or the endothelium. There were numerous small vesicles in the corneal stroma (Fig. 3a), which were of similar size to the vesicles observed with confocal microscopy. The vesicles were more prominent in the deep stroma than in the shallow stroma. Accumulations in the vesicles were Congo-red negative and alcian-blue positive (Fig. 3b). Sudan III and Sudan black staining could not confirm the presence of cholesterol ester in the vesicles. On immunohistochemical analysis, collagen VI [clone: anti-hCL (VI), mouse IgG1,к; 1:500; KYOWA PHARMA CHEMICAL CO., LTD., Toyama, Japan] was observed in some vesicles (Fig. 3c).

Since the PKP, the patient has been on regular medication with pitavastatin and tocopherol nicotinate and has had a normal lipid profile except for low serum HDL-

\section{APOA1: mRNA 897bp, Protein 267aa}

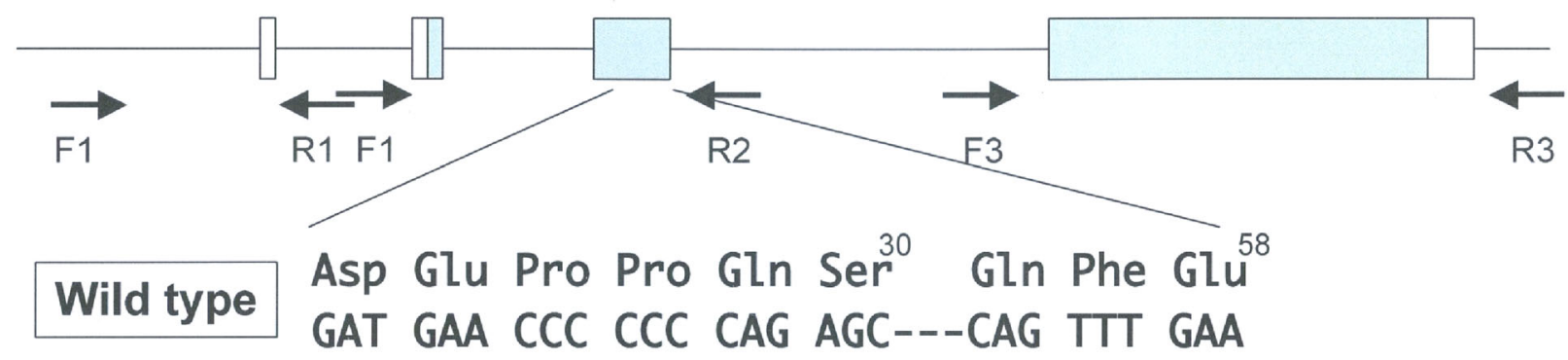

\begin{tabular}{l|lll} 
Mutant type & Asp Glu Pro Pro Gln Glu Pro Val Term \\
GAT GAA CCC CCC CCA GAG---CCA GTT TGA
\end{tabular}

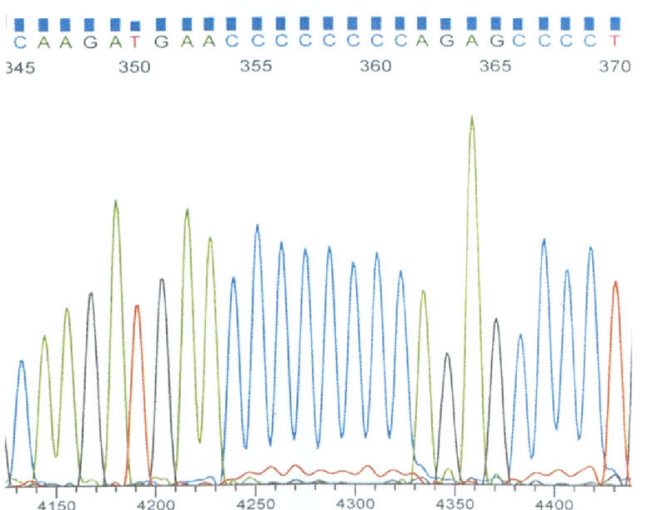

Fig. 1 The result of genetic evaluation. A single-nucleotide insertion of cytosine altered the reading frame from codon 5 and produces a premature stop codon at codon 34 


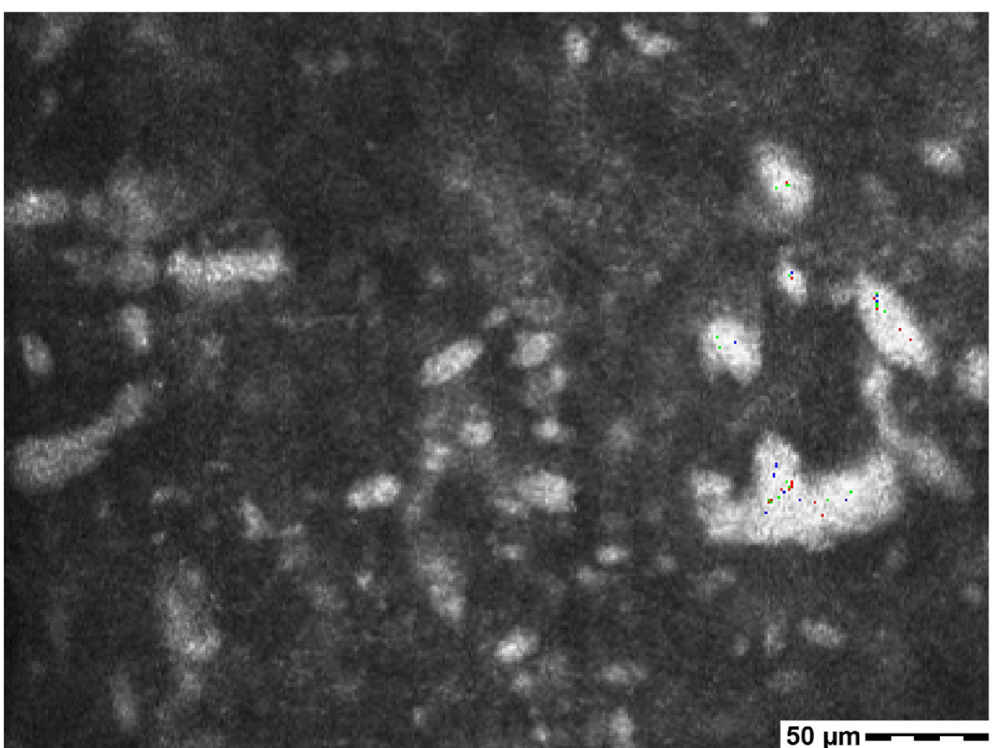

Fig. 2 Confocal microscopy in the corneal stroma. High-intensity vesicles, 10 to $100 \mu \mathrm{m}$ in diameter, were observed
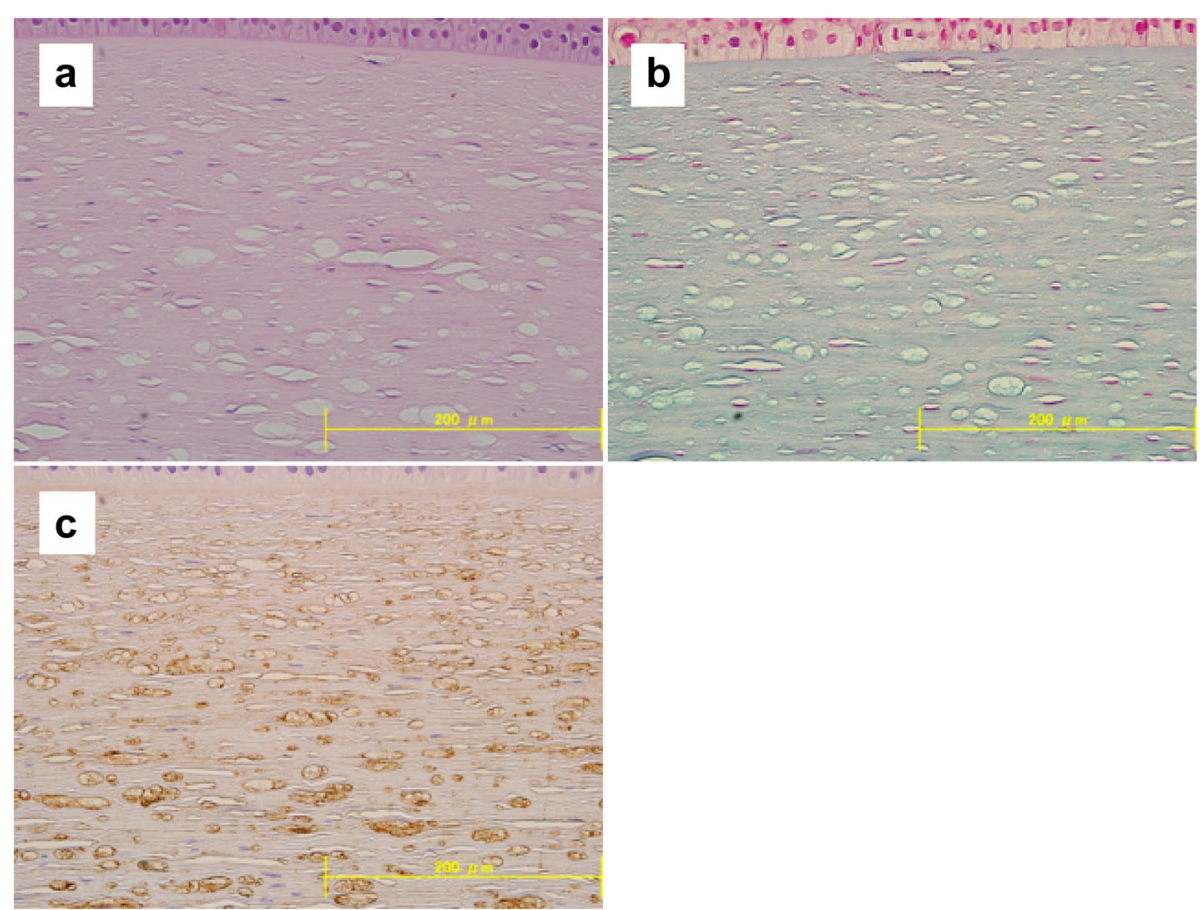

Fig. 3 Histopathology and immunohistochemistry of the surgically excised corneal button. a Histopathology of the central cornea. There were many small vesicles (10 to $100 \mu \mathrm{m}$ in diameter) the corneal stroma. The vesicles were much more numerous in the deep stroma than in the upper stroma (hematoxylin and eosin staining; magnification $\times 40$ ). $\mathbf{b}$ Histopathology of the central cornea. Accumulations in the vesicles were alcian-blue positive (alcian-blue staining; magnification $\times 40$ ). c Collagen VI immunostaining of the central cornea detected collagen VI in some of the vesicles (magnification $\times 40$ ) 
cholesterol levels. In 2015, her current visual acuity is 20/25 OS and 20/20 OD and both corneal grafts have maintained good transparency.

\section{Discussion}

Our patient demonstrated clinical findings typical to Apo A-I deficiency. Histopathologically, vesicles in the corneal stroma were significantly larger $(10-100 \mu \mathrm{m}$ in diameter) than those described in the report by Osakabe and colleagues $(200 \mathrm{~nm}-2 \mu \mathrm{m}$ in diameter) [5]. Apo A-I mutations, including Apo A-I $\mathrm{I}_{\text {Iowa }}$, are known to induce amyloidosis [6]. However, our patient did not have any accumulation of amyloid in the corneal stroma. It is possible that we failed to detect cholesterol ester in the vesicles by Sudan black staining because we embedded the corneal button in paraffin. It was also regretful that the right cornea had not been examined immunohistochemically.

Collagen VI is a major extracellular matrix protein, bridging cells to the surrounding connective tissue and organizing the three-dimensional tissue architecture. The mutations in the genes encoding Collagen VI chains result in several forms of inherited myopathies, decrease of stiffness in cartilage [7]. It is also distributed in corneal stroma, and reported the change of its expression level during wound healing period [8-10].

\section{Conclusions}

To our knowledge, ours is the first report of a patient with confirmed mucoid degeneration and accumulations of collagen VI in the stromal vesicles. Our results suggest that structural changes in corneal collagen fibrils contribute to the formation of stromal vesicles.

\section{Abbreviations}

Apo: Apolipoprotein; HDL: High-density lipoprotein; OD: Oculus Dexter; OS: Oculus Sinister; PKP: Penetrating keratoplasty

\section{Acknowledgements}

We thank Editage Author Services for editing this manuscript. We adhered to the CARE guidelines/methodology.

\section{Funding}

The case report has no funding involved.

\section{Availability of data and material}

The datasets during and/or analysed during the current study available from the corresponding author on reasonable request.

\footnotetext{
Authors' contributions

HN and MN drafted the manuscript, collected the data, and participated in the design of the study. SS and RO collected the data including genetic and histopathological evaluation. TK, MY, and HY participated in the design and critically reviewed the manuscript. All authors approved the final manuscript. All authors read and approved the final manuscript.
}

\section{Competing interests}

The authors declare that they have no competing interests.

\section{Consent for publication}

Written informed consent was obtained from the patient for publication of this case report and any accompanying images.

Ethics approval and consent to participate

Informed consent was obtained from the participant and study conduct adhered to the tenets of the Declaration of Helsinki. The study was approved by the Ethics Committee of the Yamagata University Faculty of Medicine, Yamagata, Japan.

\section{Author details}

${ }^{1}$ Department of Ophthalmology and Visual Sciences, Yamagata University Faculty of Medicine, 2-2-2 lidanishi, 9909585 Yamagata City, Yamagata, Japan. ${ }^{2}$ Department of Neurology, Hematology, Metabolism, Endocrinology and Diabetology, Yamagata University Faculty of Medicine, Yamagata City, Yamagata, Japan. ${ }^{3}$ Department of Diagnostic Pathology, Yamagata University Faculty of Medicine, Yamagata City, Yamagata, Japan.

Received: 28 September 2016 Accepted: 23 January 2017

Published online: 08 February 2017

\section{References}

1. Schaefer EJ, Ordovas JM, Law SW, Ghiselli G, Kashyap ML, Srivastava LS, Heaton WH, Albers JJ, Connor WE, Lindgren FT, et al. Familial apolipoprotein a-I and C-III deficiency, variant II. J Lipid Res. 1985;26:1089-101.

2. Ikewaki K, Matsunaga A, Han H, Watanabe H, Endo A, Tohyama J, Kuno M, Mogi J, Sugimoto K, Tada N, Sasaki J, Mochizuki S. A novel two nucleotide deletion in the apolipoprotein a-I gene, apoA-I shinbashi, associated with high density lipoprotein deficiency, corneal opacities, planar xanthomas, and premature coronary artery disease. Atherosclerosis. 2004;172:39-45.

3. Bandarian F, Hedayati M, Daneshpour MS, Naseri M, Azizi F. Genetic polymorphisms in the APOA1 gene and their relationship with serum HDL cholesterol levels. Lipids. 2013:48:1207-16.

4. Wada M, Iso T, Asztalos BF, Takama N, Nakajima T, Seta Y, Kaneko K, Taniquchi Y, Kobayashi H, Nakajima K, Schaefer EJ, Kurabayashi M. Marked high density lipoprotein deficiency due to apolipoprotein a-I tomioka (codon 138 deletion). Atherosclerosis. 2009:207:157-61.

5. Osakabe Y, Amano S, Fukuoka S, Yamagami S. Histologic evaluation of a cornea in a patient with apolipoprotein a-I deficiency. Cornea. 2008;27:372-3.

6. Nakata K, Kobayashi K, Yanagi H, Shimakura Y, Tsuchiya S, Arinami T, Hamaguchi $\mathrm{H}$. Autosomal dominant hypoalphalipoproteinemia due to a completely defective apolipoprotein a-I gene. Biochem Biophys Res Commun. 1993;129:950-5.

7. Cescon M, Gattazzo F, Chen P, Bonaldo P. Collagen VI at a glance. J Cell Sci. 2015; 128:3525-31

8. El-Shabrawi Y, Kublin CL, Cintron C. MRNA levels of alpha1(VI) collagen, alpha1(XII) collagen, and beta ig in rabbit cornea during normal development and healing. Invest Ophthalmol Vis Sci. 1998:39:36-44.

9. Koch DD. Histological changes and wound healing response following noncontact holmium: YAG laser thermal keratoplasty. Trans Am Ophthalmol Soc. 1996:94:745-802.

10. Cintron C, Hong BS. Heterogeneity of collagens in rabbit cornea: type VI collagen. Invest Ophthalmol Vis Sci. 1988;29:760-6.

Submit your next manuscript to BioMed Central and we will help you at every step:

- We accept pre-submission inquiries

- Our selector tool helps you to find the most relevant journal

- We provide round the clock customer support

- Convenient online submission

- Thorough peer review

- Inclusion in PubMed and all major indexing services

- Maximum visibility for your research 